Ingeniare. Revista chilena de ingeniería, vol. $15 \mathrm{~N}^{\circ} 1,2007, \mathrm{pp} .51-54$

\title{
CONTROL DE CALIDAD DE HERRAMIENTAS DE CORTE CON REMOCIÓN MÍNIMA DE MATERIAL
}

\section{QUALITY CONTROL OF CUTTING TOOLS WITH MINIMUM MATERIAL REMOVAL}

\author{
Jandrey Maldaner ${ }^{1} \quad$ Patrick Gleim $^{2} \quad$ Franz Tikal $^{3}$ \\ Recibido 2 de noviembre de 2005, aceptado 12 de enero de 2007 \\ Received: November 2, 2005 Accepted: January 12, 2007
}

\begin{abstract}
RESUMEN
Debido a la mejora de las tecnologías de mecanizado, es necesario que las herramientas satisfagan las demandas requeridas. Una condición especial, para poder describir con seguridad el corte con remoción mínima de material, es el conocimiento de los valores característicos de la superficie y de la geometría. Las herramientas poseen una influencia substancial en la calidad de las piezas producidas, así como en la estabilidad y en la seguridad del proceso de mecanizado. Aunque hay una gran cantidad de herramientas para el corte con remoción mínima de material, se presenta como ejemplo la investigación con medición óptica y la obtención de los valores característicos para dientes de sierras de cinta.
\end{abstract}

Palabras clave: Técnica de medición óptica, sierras de cinta, filos de corte.

\section{ABSTRACT}

Due to improved machine cutting technologies it is necessary that tools fulfil the required performance. A special condition, for minimal machine cutting removal, is the knowledge of surface and geometry characteristic values. These tools represent both a substantial influence on the quality of the manufactured pieces and on stability and working safety of the cutting process. Since there is a large number of tools for minimal removal of material, examples of optical measurement and examination of characteristic values by the belt saw teeth are presented.

Keywords: Optical technology of measurement, saw band, cutting edge.

\section{INTRODUCCIÓN}

Con el objetivo de aumentar la vida útil de las sierras de cinta y para ofrecer una mejor calidad de corte, el Institut für Produktionstechnik und Logistik (IPL) de la Universidad de Kassel (Alemania), en sociedad con el Bremer Institut für angewandte Strahltechnik (BIAS), la compañía Roth \& Rau (desarrollo de tecnología de plasma) y el fabricante de sierras de cinta WIKUS desarrollarán el proyecto de Plasma-fotónico, que prevé la introducción de revestimientos superficiales de diamante en las puntas de metal duro de los dientes de las sierras. La aplicación es realizada por un proceso de revestimiento superficial de deposición química por vapor (CVD) enriquecido por plasma.
Los investigadores involucrados en el proyecto creen que el resultado de esta inversión se traducirá también en una economía de costos y el aumento de la protección al ambiente, una vez que sea posible utilizar una menor cantidad de líquidos lubricantes y refrigerantes en el corte con sierra.

El Institut für Produktionstechnik und Logistik realiza la medición y el análisis de los filos de corte antes y después del revestimiento superficial de los dientes de sierra, asimismo la investigación del espesor del revestimiento, el cual es del orden de algunos micrones. Otra área implicada, son las técnicas de producción y de mecanizado, también en la Universidad de Kassel, la cual realiza investigaciones y desarrollos de nuevas geometrías de herramientas para materiales de difícil mecanizado.

\footnotetext{
1 Institut für Produktionstechnik und Logistik, Universität Kassel / FB15, Kurt-Wolters-Straße 3, 34125 Kassel - Alemania, maldaner@uni-kassel.de

2 Institut für Produktionstechnik und Logistik, Universität Kassel / FB15, Kurt-Wolters-Straße 3, 34125 Kassel - Alemania, pgleim@uni-kassel.de

3 Institut für Produktionstechnik und Logistik, Universität Kassel / FB15, Kurt-Wolters-Straße 3, 34125 Kassel - Alemania, tikal@uni-kassel.de
} 


\section{PROCESO DE MEDICIÓN}

Para buscar las características especiales en el mecanizado con geometrías definidas de corte (como el típico proceso de torneado) con remoción mínima de material, así como en el proceso de corte con sierra, es necesario conocer la geometría del diente de sierra, es decir, la topografía de la superficie del filo de corte y la relación del redondeo del filo de corte con el espesor de corte.

A través de la microscopía electrónica de barrido (SEM) y el espectrómetro de Raman, pueden ser obtenidos resultados cualitativos sobre la constitución química y la estructura del revestimiento superficial, mientras que los resultados cuantitativos, sobre dimensiones y formas, son conseguidos principalmente por técnica de medición sin contacto (óptico) en 3D.

Las nuevas técnicas de la medición óptica tridimensional, entre ellas la de aberración cromática, hacen posible medir geometrías y microtopografías de superficie con alta resolución lateral. Los resultados obtenidos por la técnica de aberración cromática son comparables con los de medición con contacto (táctil), pero es un proceso más lento. Entre los sistemas ópticos de medición se incluyen el interferómetro de líneas blancas, el microscopio de fuerza atómica, la proyección de rayos, la técnica confocal por láser y la técnica del autofoco por láser.

Estos sistemas se pueden utilizar en equipamientos automatizados con interfaz Windows, para superficies suaves, para perfiles con profundidades pequeñas y para mediciones no-destructivas.

\section{SISTEMA DE MEDICIÓN}

En sus investigaciones, el IPL utiliza el dispositivo de medición MicroProf ${ }^{\circledR}$, figura 1, producido por la compañía alemana Fries Research \& Technology (FRT), para la metrología de superficies.

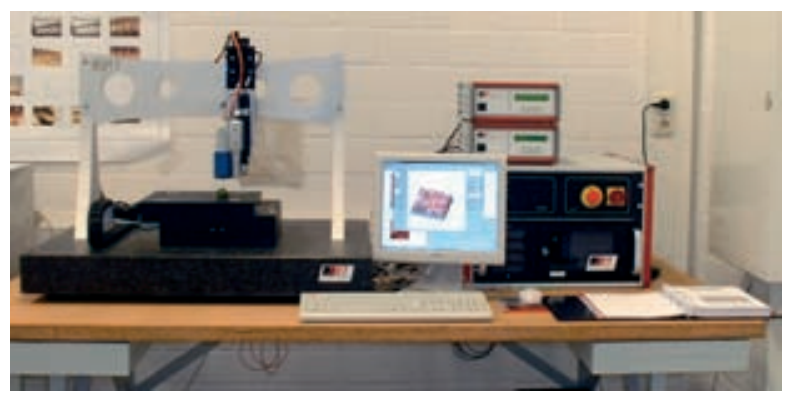

Figura 1. Equipo de medición óptica Microprof ${ }^{\circledR}$.
Los sensores utilizados por dicho equipo operan de acuerdo con el principio de la aberración cromática, por lo cual una luz blanca es focalizada en el componente y la altura del objeto a ser medido es indicada por los sensores como una área marcada, a partir de la distribución espectral de la luz que es disipada en la superficie, figura 2.

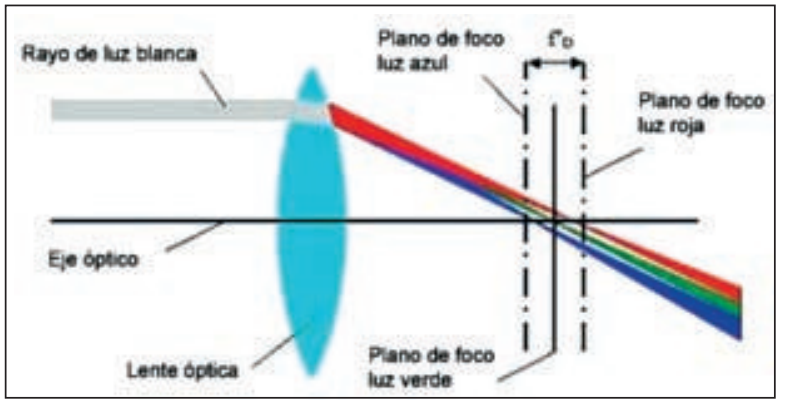

Figura 2. Principio de aberración cromática.

Entonces, es posible medir contornos, rugosidades y topografías de superficie, y determinar el espesor de revestimientos superficiales, de acuerdo con la tarea de ser efectuada. Esto ocurre sin contacto y es aplicable en todos los tipos de superficies: transparentes, especulares o negras, sin dejar marcas de medición.

En el principio de aberración cromática, una cantidad pequeña de luz es reflejada por el objeto que se medirá, para garantizar la medida exacta. Y como los sensores trabajan sin un control activo, es posible realizar mediciones rápidas en piezas complejas.

El trabajo se hace en conjunto con un software de control y otro de análisis. Para tareas rutinarias, existe la posibilidad de escribir programas para automatizar las mediciones.

\section{MEDIDA DE LA FORMA DE DIENTES DE SIERRAS DE CINTA}

El corte por sierras de cinta, usado en materiales sólidos, tubulares, estructurales y de piezas fundidas, es realizado a través de diversas formas y modelos de dientes. Un punto esencial en la medición de los dientes de sierras de cinta es la determinación cualitativa y cuantitativa de la forma y la dimensión del redondeo de los filos de corte.

Como características principales de este proceso de corte, se pueden mencionar los filos de corte y sus geometrías de dimensiones micrométricas, además de las superficies, los filos y los ángulos de corte. Los filos de corte y su estado de desgaste tienen influencia predominante en el 
proceso de corte. Por lo tanto, es importante la medición de sus puntas. Los filos de corte exigen un redondeo de precisión, debido al perfil del esfuerzo actuante con sus altos componentes dinámicos y la alta velocidad de carga sobre el revestimiento superficial en el transcurso del proceso. Con un redondeo acentuado, solamente el área del redondeo de la punta del filo será capaz de mecanizar el material. Esto conduce a un aumento indebido de la fuerza de corte y al desgaste de la herramienta.

El uso de las sierras de cinta con dientes de metal duro presenta una alta tasa de crecimiento, debido a su gran capacidad de producción. Con este material se obtiene una mejor relación entre eficacia económica y la calidad de la técnica del corte.

El proceso del corte con sierra de alta capacidad se logra mediante dientes con ángulos de corte reducidos y ángulos de salidas más grandes. Las fuerzas originadas en el proceso de corte, en especial en el corte de materiales de difícil mecanizado, llevan fácilmente a la rotura y astillamiento de los filos de corte, principalmente en procesos inestables. Para proteger y aumentar la capacidad de este metal de corte relativamente frágil se utilizan chaflanes de protección y/o de los respectivos redondeos.

\section{RESULTADOS DE MEDICIÓN EN DIENTES DE SIERRAS DE CINTA}

Para alcanzar resultados en lo referente al redondeo de la punta de los filos de corte se efectúa una medición con un sensor, que tiene una amplitud de medición máxima de 3 [mm] y una resolución vertical de 30 [nm]. La geometría del diente de sierra es proyectada y desarrollada en un área alrededor del filo de corte. La medición fue efectuada en una área de $1\left[\mathrm{~mm}^{2}\right]$, figura 3.

Con la ayuda del software de análisis se obtienen perfiles del diente de sierra, a partir de los datos de la medición anterior hecha en 3D. Ajustando una curva sobre el perfil del diente de sierra fue posible medir el radio de redondeo de la punta del filo de corte de 18 [ $\mu \mathrm{m}]$, figura 4.

En lo referente a los valores obtenidos a través de la medición táctil, es posible apreciar también los diferentes parámetros de rugosidad. Hasta antes el desarrollo de la tecnología óptica de medición, parámetros como $\mathrm{R}_{Z}$ o $\mathrm{R}_{\text {máx }}$ eran determinados a partir de un perfil de una línea recorrida sobre el objeto.

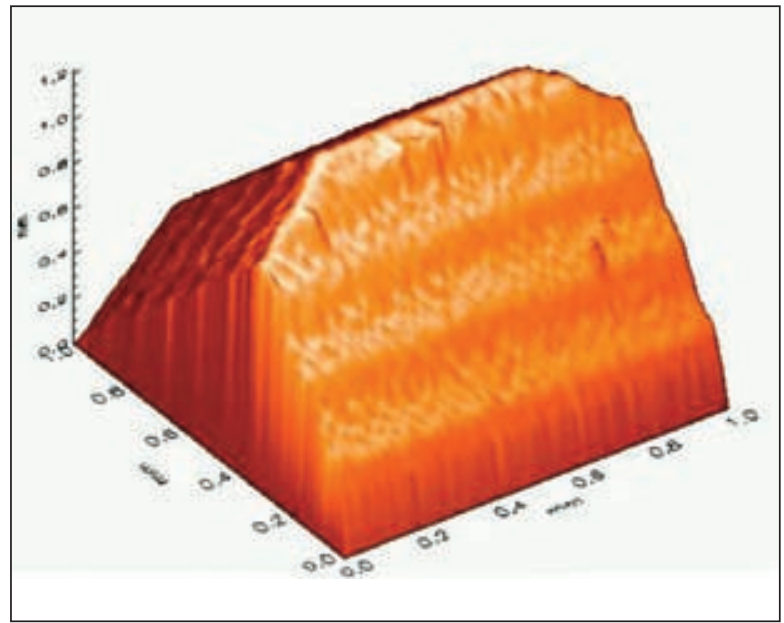

Figura 3. Representación de la topografía superficial $3 \mathrm{D}$ de un diente de sierra.

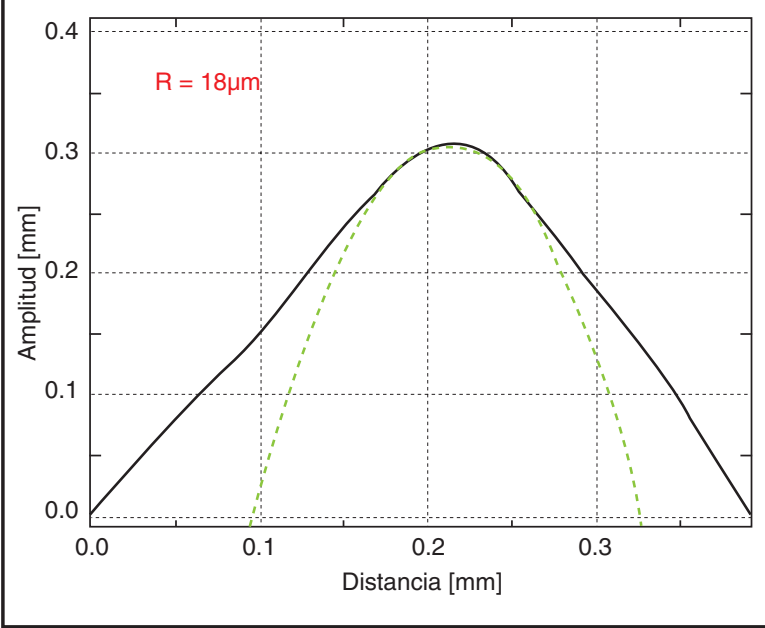

Figura 4. Corte del perfil obtenido por medición topográfica 3D de un diente de sierra.

\section{CONCLUSIÓN}

La técnica de medición óptica permite, mediante su utilización, una caracterización sin contacto y nodestructiva de microestructuras sin un alto consumo de tiempo con la preparación de las probetas. El método de medición es apropiado especialmente en procesos, en los cuales debe ser efectuado un análisis de superficie (2D) y un examen del perfil (3D). El equipamiento complementa los demás instrumentos de medición de superficies por contacto existentes en el Instituto de Técnicas de Producción y Logística. A través de los softwares existentes es posible la obtención y el análisis rápido de topografías de superficies $3 \mathrm{D}$. 


\section{REFERENCIAS}

[1] H. Tschätsch. "Praxis der Zerspantechnik". Vieweg-Verlag. Braunschweig / Wiesbaden. 2002.
[2] Norma ISO 4875. "Bandsägeblätter für Metall”. Teil 1. Teil 2 und Teil 3. 2006.

[3] Material informativo de la empresa FRT GmbH. Bergisch Gladbach. 2004. 